\title{
Evaluation of Image Signal-to-Noise Ratio in Time-of-flight PET
}

\author{
Enrico Clementel Student Member, IEEE, Stefaan Vandenberghe, Joel S.Karp Senior Member, IEEE, \\ Suleman Surti Senior Member, IEEE
}

\begin{abstract}
In PET imaging Noise Equivalent Counts (NEC) is a common image quality index, derived to be proportional to image $\mathrm{SNR}^{2}$ and used as an index of general system performance. Many studies have shown that TOF information leads to reduced noise, faster image convergence, and improved SNR. However, the original NEC formula does not account for any contribution of timing resolution to the final image quality, and modified versions of the formula have been proposed to account for the reduction in noise variance and increased sensitivity due to TOF information. In this study, we aim to investigate the relationship between NEC and image SNR in uniform phantoms when OSEM and TOF OSEM image reconstruction is applied. Two cylindrical uniform phantoms, 20 and $35 \mathrm{~cm}$ in diameter, were acquired over a wide range of activity levels on a Philips Gemini TF PET scanner. Multiple realizations of the original scans were obtained through bootstrapping and reconstructed with OSEM and TOF OSEM algorithms to obtain mean and standard deviation images. The ratio of the mean value in a central ROI over both images was taken as a measure of image SNR. NEC was calculated from the original data using both the classical and a TOF-adapted formula. The results show that Trues have a better proportionality with image $\mathrm{SNR}^{2}$ than NEC and TOF NEC in the considered range of activities. Timing resolution and Random fraction appear to have a limited influence on image SNR, but the usage of a matched TOF kernel in reconstruction is found to be necessary to maximize the gain.
\end{abstract}

\section{INTRODUCTION}

$\mathbf{N}$ OISE Equivalent Counts (NEC) is a common image quality index in PET, derived by Strother [1] to be proportional to the square of image Signal-to-Noise Ratio (SNR) and is used as an index of general system performance. It is given by:

$$
N E C=\frac{T^{2}}{T+S+\frac{D}{D_{F O V}} \cdot R}
$$

where $\mathrm{T}$ represents the Trues, $\mathrm{S}$ the Scatter and $\mathrm{R}$ the Random coincidences, D represents the object diameter, $\mathrm{D}_{F O V}$ the active Field-of-View diameter.

As clinical Time-of-Flight (TOF) PET scanners are now

Manuscript received November 10, 2010.

E. Clementel and S. Vandenberghe are with the Department of Electronics and Information Systems, MEDISIP, Ghent University-IBBT-IBiTech, De Pintelaan 185 block B, B-9000 Ghent, Belgium (telephone: +39-9-3324322, e-mail: enrico.clementel@ugent.be).

S. Surti is with the Department of Radiology of the University of Pennsylvania, 404 Blockley Hall, 425 Guardian Drive, Philadelphia, PA 19104 USA (telephone: 215-662-7214, e-mail: surti@ mail.med.upenn.edu).

Joel S. Karp is with the Department of Physics and Astronomy and the Department of Radiology of the University of Pennsylvania, 404 Blockley Hall, 425 Guardian Drive, Philadelphia, PA 19104 USA (telephone: 215-6623073, e-mail: joelkarp@mail.med.upenn.edu). available, many studies have shown that TOF information leads to reduced noise, faster image convergence, and improved SNR [2], [3], [4], [5], [6], [7], [8], [9], [10], [11]. The original NEC formula does not account for any contribution of timing resolution to the final image quality. A formula incorporating the effects of TOF information (reduction in noise variance and increased sensitivity, [12], [13]) was proposed by Conti [14]:

$$
\text { TOF NEC }=\frac{D}{\Delta x} \cdot \frac{T^{2}}{T+S+\left(\frac{D}{D_{F O V}}\right)^{2} \cdot R}
$$

where $\Delta x$ represents the positioning uncertainty determined by the systems timing resolution.

At first approximation, based on formulas (1) and (2), the TOF SNR gain due to increased sensitivity is predicted to be proportional to the object dimension:

$$
\text { TOF SRN Gain } \propto \frac{D}{\Delta x}
$$

the aim of our study is to investigate the relationship between NEC and image SNR in uniform phantoms when OSEM and TOF OSEM image reconstruction is applied, and to study the influence of timing resolution and Random Fraction on image SNR.

\section{Materials And Methods}

\section{A. Data Acquisition}

Two cylindrical phantoms, $30 \mathrm{~cm}$ long, 20 and $35 \mathrm{~cm}$ in diameter, were filled with a uniform solution of FDG and water. The initial activity concentrations were $6.216 \mathrm{kBq} / \mathrm{ml}$ $(0.168 \mu \mathrm{Ci} / \mathrm{ml})$ for the $35 \mathrm{~cm}$ phantom and $22.2 \mathrm{kBq} / \mathrm{ml}(0.60$ $\mu \mathrm{Ci} / \mathrm{ml}$ ) for the $20 \mathrm{~cm}$ phantom. Multiple scans were acquired in list mode format during activity decay and final activity concentrations were $0.407 \mathrm{kBq} / \mathrm{ml}(0.014 \mu \mathrm{Ci} / \mathrm{ml})$ for the 35 $\mathrm{cm}$ phantom and $0.518 \mathrm{kBq} / \mathrm{ml}(0.011 \mu \mathrm{Ci} / \mathrm{ml})$ for the $20 \mathrm{~cm}$ phantom. Timing resolution varied between 610 and 800 ps in the considered range of activities due to degradation effects occurring at high count rates in our scanner, a Philips Gemini TF [2].

\section{B. List mode data generation}

Different list mode data were considered in this study:

Original data: The original datasets acquired on the scanner at varying count rate for each phantom.

Frac $_{800}$ : A high count rate list mode dataset, for which the original timing resolution was $800 \mathrm{ps,} \mathrm{was}$ 
fractionated in 5 smaller datasets containing $12 \%$, $26 \%, 38 \%, 57 \%, 78 \%$ of the original dataset's True coincidences. The data were reconstructed with TOF OSEM with a matching 800 ps TOF kernel, as well as with non-TOF OSEM. These additional datasets were generated with the purpose of comparison of a high count rate dataset with increased Random fraction and Scatter fraction, worse timing resolution but short scan time with lower activity data containing an equal amount of Trues but better timing resolution and lower Random fraction and Scatter fraction.

Frac $_{610}$ : The same fractionated data from above $\left(\mathrm{Frac}_{800}\right)$ were reconstructed with the intrinsic TOF kernel of the scanner $(610 \mathrm{ps})$, to investigate the effect of ignoring the degrading effects of timing resolution occurring at high count rates.

Degr $_{800}$ : The timing resolution of 5 selected original datasets was degraded via software to 800 ps and reconstructed with a matching TOF kernel. Timing resolution for the original data points was 610,640 , 660,700 and $750 \mathrm{ps}$. These data were generated for comparison with the original data, which have identical Random fraction, Scatter fraction and scan time but better timing resolution.

\section{Bootstrapping}

Multiple realizations of each list dataset were obtained following Dahlbom's bootstrap approach [15]. The optimal number of bootstrapped realizations was determined prior to the study: 120 bootstrapped copies of the $35 \mathrm{~cm}$ phantom data set were generated and reconstructed, and 12 Standard Deviation images SD were calculated using 10, 20, ...,120 of the bootstrapped copies. The sum of all pixels in each SD image was taken as a figure of merit representing the total SD, and its convergence was taken as an indication of the minimum number of bootstrapped datasets necessary to accurately replicate the noise statistic of the original dataset. Based on this study we chose to bootstrap 60 copies per each dataset, as a good convergence of SD values is obtained for both TOF and non-TOF images.

\section{Image reconstruction algorithm}

An OSEM and TOF OSEM list mode algorithm with 33 subsets was used for image reconstruction. Corrections for attenuation (CT based), detector efficiency/normalization, Scatter (TOF Single Scatter Simulation [16], [17]), and Random coincidences (Delayed Coincidence Window) were incorporated in the system model. To determine the number of iterations to be used in the study, both phantoms, containing a set of six $1 \mathrm{~cm}$ spheres, were filled with a total activity of $111 \mathrm{MBq}$ and an 8:1 sphere-to-background ratio. Based on the Contrast Recovery Coefficient curves, 3 and 6 iterations respectively for TOF and non-TOF image reconstruction were chosen for the $20 \mathrm{~cm}$ phantom, and 2 and 5 iterations for TOF and non-TOF were chosen for the $35 \mathrm{~cm}$ phantom, in order to achieve similar contrast values close to convergence. The

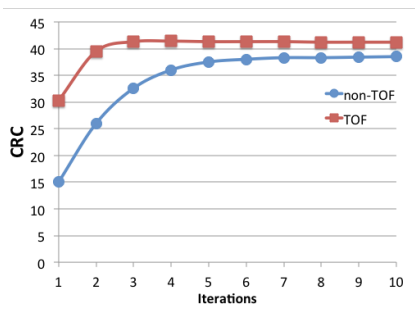

(a)

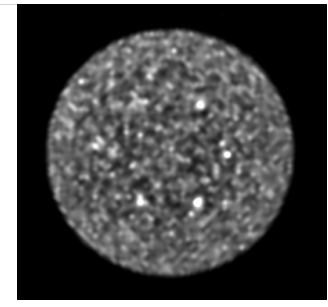

(b)
Fig. 1: (a) Contrast Recovery Coefficient curves for the $35 \mathrm{~cm}$ phantom for TOF, in red, and non-TOF reconstruction, in blue; (b) corresponding image. Four of the hot lesions are visible.

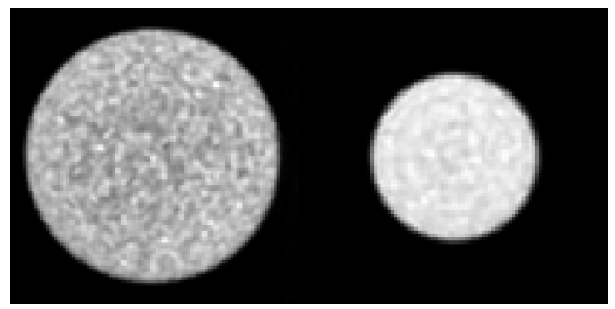

(a)

(b)

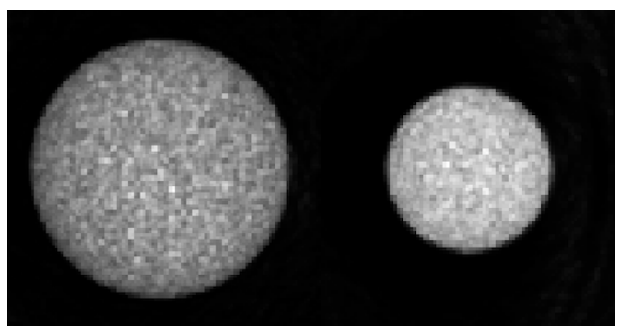

(c)

(d)

Fig. 2: Example of mean (top) and Standard Deviation (bottom) for the $35 \mathrm{~cm}$ phantom (left) and the $20 \mathrm{~cm}$ phantom (right)

CRC curve and a transaxial image of the 35 phantom used for its calculation is shown in Figure 1.

\section{E. SNR measurement and NEC calculation}

For each dataset of the original and additional data, Mean and Standard Deviation (SD) images were obtained from the 60 bootstrap realizations; an example of such images is shown in Figure 2: visual assessment shows that a good level of uniformity is achieved for both phantoms indicating accurate corrections for Random and Scatter coincidences. SNR was measured as the ratio between the mean values in a $4 \mathrm{~cm}$ ROI centered on both images. TOF SNR Gain was also calculated as the ratio:

$$
\text { Gain }=\frac{T O F S N R^{2}}{\text { nonTOF } S N R^{2}}
$$

\section{RESULTS AND DISCUSSION}

Figure 3 shows the $\mathrm{SNR}^{2}$ vs NEC and $\mathrm{SNR}^{2}$ vs Trues plot for the $20 \mathrm{~cm}$ phantom. A linear correlation is clear in both plots for the original data and for the $\mathrm{Frac}_{800}$ data. Figure 

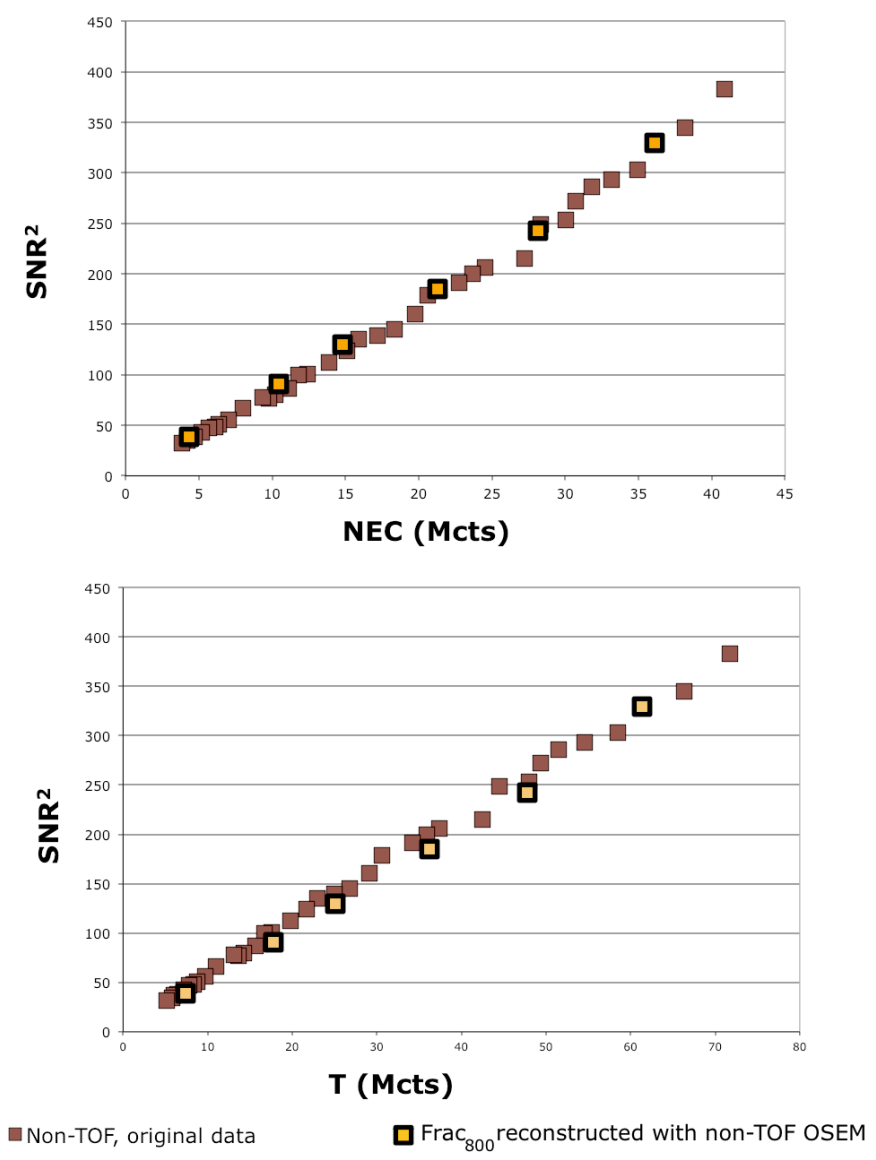

Fig. 3: Results for the $20 \mathrm{~cm}$ phantom images reconstructed without TOF information; brown squares: original data, orange squares: $\mathrm{Frac}_{800}$ data.

Top: image $\mathrm{SNR}^{2}$ is plotted against NEC, calculated with formula 1; bottom: image $\mathrm{SNR}^{2}$ is plotted against Trues.

4 shows the corresponding plots for the $35 \mathrm{~cm}$ phantom. $\mathrm{SNR}^{2}$ shows a non-linear behavior against NEC. In particular, $\mathrm{Frac}_{800}$ data, which contains a high fraction of Randoms, has the same NEC as a corresponding original dataset but higher $\mathrm{SNR}^{2}$. The correlation of image $\mathrm{SNR}^{2}$ with Trues, however, is still linear. This suggests that randoms variance do not significantly affect image SNR and that the NEC formula seems to overestimate noise propagation when a blob-based OSEM reconstruction algorithm is applied, as it potentially better controls noise propagation. This is clearly observed in the larger object but is not apparent for the data of the smaller one: in our experimental setup, at the highest activity level and considering the size of the $20 \mathrm{~cm}$ diameter phantom, the randoms fraction is limited to $30 \%$, and noise propagation is therefore also limited. NEC and Trues do not differ significantly and both show a good relationship with image $\mathrm{SNR}^{2}$. In the case of the $35 \mathrm{~cm}$ phantom data, however, we observe a considerably higher randoms fraction of $70 \%$ at the highest activity point. In the latter case, noise propagation could potentially have a larger effect on the final image and the differences between $\mathrm{SNR}^{2}$ as predicted by NEC and $\mathrm{SNR}^{2}$ as predicted by Trues are clearly visible.
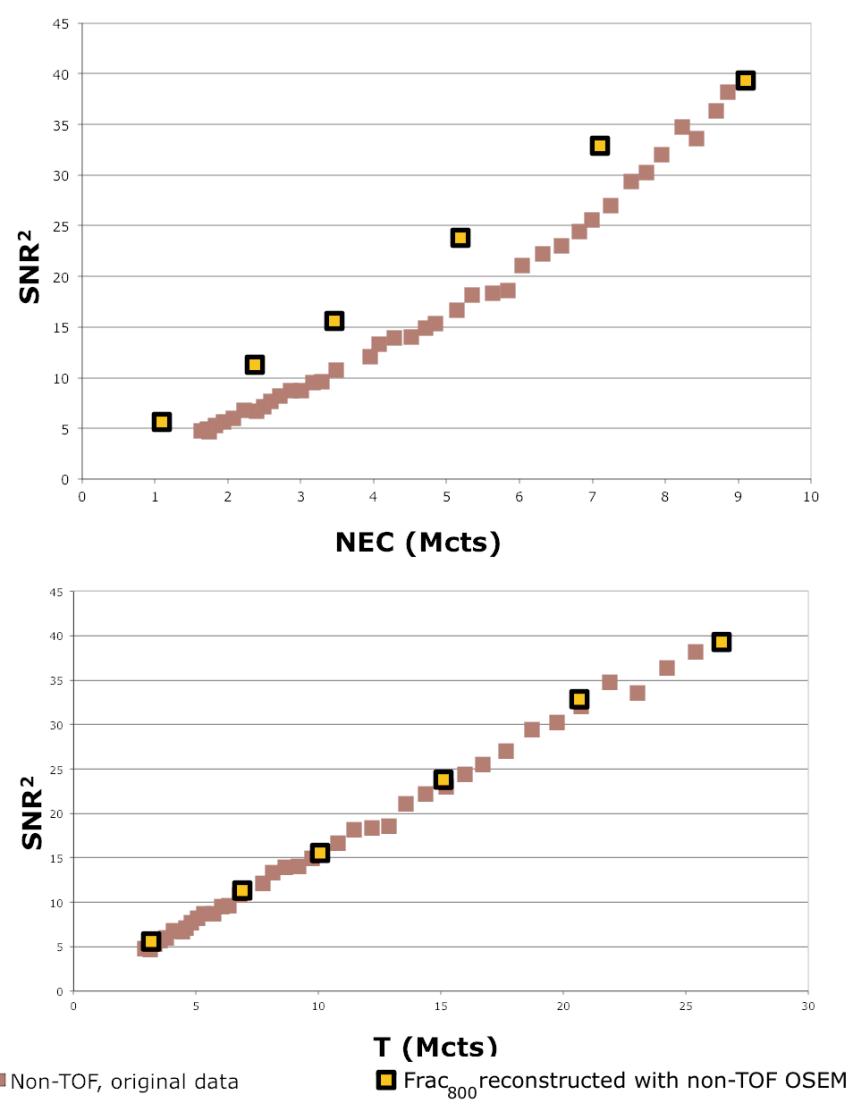

Fig. 4: Results for the $35 \mathrm{~cm}$ phantom images reconstructed without TOF information; brown squares: original data, orange squares: $\mathrm{Frac}_{800}$ data.

Top: image $\mathrm{SNR}^{2}$ is plotted against NEC, calculated with formula 1; bottom: image $\mathrm{SNR}^{2}$ is plotted against Trues.

Figure 5 shows the plot for TOF $\mathrm{SNR}^{2}$ vs Trues for both phantoms, and non-TOF $\mathrm{SNR}^{2}$ for visual reference. A good proportionality is observed for the original data for both objects. Degr 800 data line up well with the original data, suggesting timing resolution influence on image SNR is limited in the considered activity range. Frac 800 also lines up with the original data. As these data have a comparable amount of Trues but a higher Random fraction than the corresponding underlying original data point, this suggests that the Random fraction influence on image SNR is also limited in the considered activity range. Frac 610 proportionality with the Trues is accurate but with a shallower slope than the original data reconstructed with a matching TOF kernel, suggesting the use of a matched TOF kernel is beneficial in terms of image SNR. The measured TOF SNR Gain is 2.1 for the $35 \mathrm{~cm}$ phantom and 1.5 for the $20 \mathrm{~cm}$ phantom. The ratio of the two Gain measures is 1.4 , which is lower than the ratio of the two diameters (1.75).

Figure 6 shows the plot for TOF $\mathrm{SNR}^{2}$ vs TOF NEC calculated according to the formula in (2). No proportionality is observed between TOF SNR ${ }^{2}$ and TOF NEC for the original data on both phantoms. This is more clearly visible for 

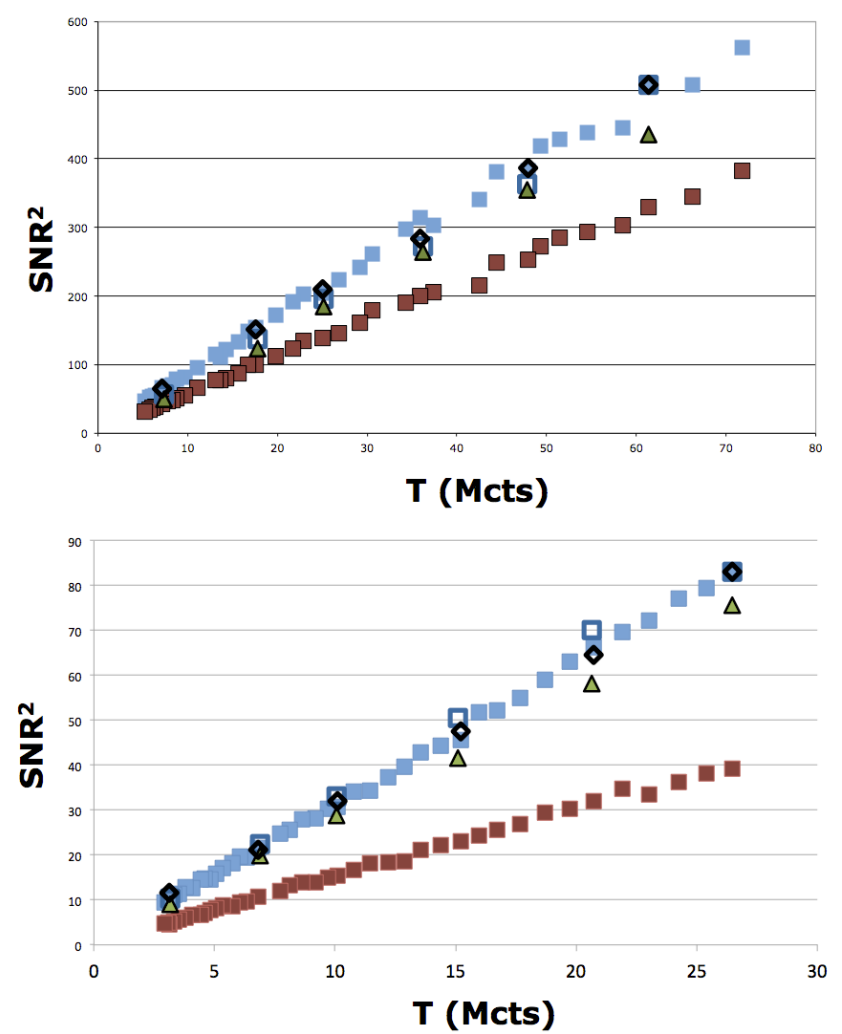

-TOF, original data $\diamond$ Degr800 QFrac800 $\Delta$ Frac610 $\square$ Non-TOF, original data

Fig. 5: Top: $\mathrm{SNR}^{2}$ vs Trues for the $20 \mathrm{~cm}$ phantom data, reconstructed with TOF OSEM; Bottom: $\mathrm{SNR}^{2}$ vs Trues for the $35 \mathrm{~cm}$ phantom data, reconstructed with TOF OSEM;

$35 \mathrm{~cm}$ phantom. Degr 800 and $\mathrm{Frac}_{800}$ data for the $20 \mathrm{~cm}$ phantom follow a similar trend as the original data. Frac $_{610}$ data for the $20 \mathrm{~cm}$ phantom shows a similar trend but with a shallower slope, indicating lower SNR than the corresponding original data with same TOF NEC. Frac 800 data for the 35 $\mathrm{cm}$ phantom show higher image $\mathrm{SNR}^{2}$ than the corresponding underlying original data having the same TOF NEC. The same consideration applies to $\mathrm{Degr}_{800}$. $\mathrm{Frac}_{610}$ data follows a similar trend as the original data, has the same TOF NEC but lower SNR as the corresponding original data.

\section{CONCLuSIONS}

Under our experimental conditions, Trues are found to have a better proportionality with image $\mathrm{SNR}^{2}$ than NEC with both TOF and non-TOF OSEM image reconstruction in the considered range of activities. TOF SNR ${ }^{2}$ gain is larger for the larger cylinder but does not increase linearly with the diameter as predicted by equation (3).

Figure 5 clearly shows the advantage in terms of image SNR when using TOF, as pointed by the steeper slope of the TOF reconstructed data line. However, as the additional data line up with the original data, the influence of timing resolution and Random fraction on image SNR seems to be limited in the ranges considered. The systematically shallower slope of the Frac 610 data indicates that the use of matched TOF kernel during reconstruction is required to maximize the gain.
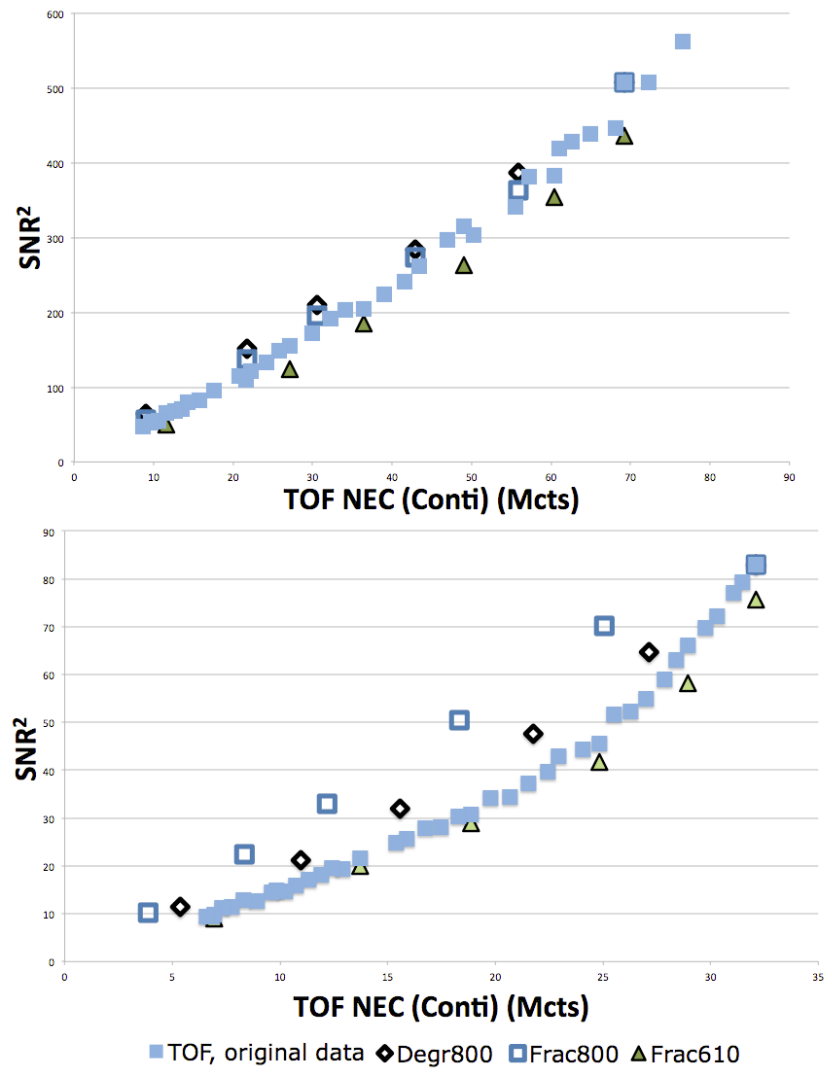

Fig. 6: Top: $\mathrm{SNR}^{2}$ vs TOF NEC for the $20 \mathrm{~cm}$ phantom data, reconstructed with TOF OSEM; Bottom: SNR $^{2}$ vs TOF NEC for the $35 \mathrm{~cm}$ phantom data, reconstructed with TOF OSEM;

These results are focused on image pixel SNR and reflect the influence of TOF on the image pixel SNR only. We do not consider the improvements in lesion contrast and detectability that have been previously associated with TOF image reconstruction.

For the purpose of this study we choose a constant iteration number for TOF and non-TOF image reconstruction for varying count levels and count statistics. Although we expect data to require a different number of iterations to achieve contrast convergence at different count rates and count statistics, calculating the optimal number of iterations for each of the datasets would be impractical; moreover, the optimal number of iterations would depend not only on count rate, but also on the image quality index considered for convergence, as lesion detectability and contrast recovery usually converge at different iterations number. Our setup choice provides similar CRC values for TOF and non-TOF images and corresponds to the clinical approach of setting imaging reconstruction parameters to fixed values.

\section{ACKNOWLEDGMENT}

The authors would like to thank Matt Werner for his support and insight on image reconstruction, and Randy Kulp for his help with data acquisition. The authors would also like to acknowledge the NIH grants R01-CA113941 (J S Karp) and R01-EB009056 (S Surti). 


\section{REFERENCES}

[1] S. Strother, M. Casey, and E. Hoffman, "Measuring PET scanner sensitivity: relating countrates to image signal-to-noise ratios using noise equivalents counts," Nuclear Science, IEEE Transactions on, vol. 37, no. 2, pp. 783-788, 2002.

[2] S. Surti, A. Kuhn, M. E. Werner, A. Perkins, J. Kolthammer, and J. S. Karp, "Performance of Philips Gemini TF PET/CT scanner with special consideration for its time-of-flight imaging capabilities," Journal of Nuclear Medicine, vol. 48, no. 3, pp. 471-80, march 2007.

[3] B. Jakoby, Y. Bercier, M. Conti, M. Casey, T. Gremillion, C. Hayden, B. Bendriem, and D. Townsend, "Performance investigation of a timeof-flight PET/CT scanner," in Nuclear Science Symposium Conference Record, 2008. NSS'08. IEEE. IEEE, 2009, pp. 3738-3743.

[4] B. Kemp, J. Williams, R. Ruter, V. Lowe, and B. Mullan, "Performance measurements of a whole body PET/CT system with time-of-flight capability," in Society of Nuclear Medicine Annual Meeting Abstracts, vol. 50, no. Supplement 2. Soc Nuclear Med, 2009, p. 1546.

[5] S. Surti, S. Karp, L. Popescu, E. Daube-Witherspoon, and M. Werner, "Investigation of time-of-flight benefit for fully 3-DPET," Medical Imaging, IEEE Transactions on, vol. 25, no. 5, pp. 529-538, 2006.

[6] M. Conti, B. Bendriem, M. Casey, M. Chen, F. Kehren, C. Michel, and V. Panin, "First experimental results of time-of-flight reconstruction on an LSO PET scanner," Physics in Medicine and Biology, vol. 50, p. 4507, 2005.

[7] S. Vandenberghe, J. S. Karp, and I. Lemahieu, "Influence of TOF resolution on object dependent convergence in iterative reconstruction," Journal of Nuclear Medicine, vol. 47, p. 58P, 2006.

[8] J. S. Karp, S. Surti, M. E. Daube-Whiterspoon, and G. Muehllehner, "Benefit of Time-of-Flight in PET: Experimental and clinical results," Journal of Nuclear Medicine, vol. 49, no. 3, pp. 462-470, march 2008.

[9] C. Lois, B. Jakoby, M. Long, K. Hubner, D. Barker, M. Casey, M. Conti, V. Panin, D. Kadrmas, and D. Townsend, "An assessment of the impact of incorporating time-of-flight information into clinical PET/CT imaging," Journal of Nuclear Medicine, vol. 51, no. 2, p. 237, 2010.

[10] S. Surti and J. Karp, "Experimental evaluation of a simple lesion detection task with time-of-flight PET," Physics in medicine and biology, vol. 54, p. 373, 2009.

[11] D. Kadrmas, M. Casey, M. Conti, B. Jakoby, C. Lois, and D. Townsend, "Impact of time-of-flight on PET tumor detection," Journal of Nuclear Medicine, vol. 50, no. 8, p. 1315, 2009.

[12] T. Budinger, "Time-of-flight positron emission tomography: status relative to conventional PET," Journal of nuclear medicine, vol. 24, no. 1, p. 73, 1983.

[13] T. Tomitani, "Image reconstruction and noise evaluation in photon timeof-flight assisted positron emission tomography," Nuclear Science, IEEE Transactions on, vol. 28, no. 6, pp. 4581-4589, 2007.

[14] M. Conti, "Effects of randoms on signal-to-noise-ratio in TOF PET," IEEE Nuclear Science Symposium Medical Imaging Conference Record, vol. 1, pp. 1590-1595, 2005.

[15] M. Dahlbom, "Estimation of image noise in pet using the bootstrap method," IEEE Trans Nucl Sci, vol. 49, pp. 2062-2066, 2002.

[16] C. C. Watson, "Extension of Single Scatter Simulation to Scatter Correction of Time-of-flight PET," IEEE Transactions on Nuclear Science, vol. 54, no. 5, pp. 1679-86, October 2007.

[17] M. E. Werner, S. Surti, and J. S. Karp, "Implementation and Evaluation of a 3D PET Single Scatter Simulation with TOF Modeling," IEEE NSS Conference Record, vol. 1, pp. 1768-1773, 2006. 